\title{
COVID-19 and diabetes: How to manage?
}

\author{
Maskey R ${ }^{1}$, Gupta $\mathrm{PP}^{2}$ \\ ${ }^{1}$ Department of Internal Medicine, BPKIHS, ${ }^{2}$ Department of General Practice and Emergency \\ Medicine
}

\section{INTRODUCTION}

Patients with diabetes have an increased risk of severe complications including Adult Respiratory Distress Syndrome and multiorgan failure. Depending on the global region, $20-50 \%$ of patients in the coronavirus disease 2019 (COVID-19) pandemic had diabetes.

The worse outcomes is seen in people with diabetes, cardiovascular disease, and hypertension. ${ }^{1}$ COVID-19 is associated with hyperglycaemia particularly in the elderly with type 2 diabetes and increased risk of infection because of defects in innate immunity affecting phagocytosis, neutrophil chemotaxis, and cell-mediated immunity.

Angiotensin-converting-enzyme 2 (ACE2) has been identified as the receptor for the coronavirus spike protein and has protective effects regarding inflammation. Acute hyperglycaemia upregulate ACE2 expression on cells to facilitate viral cell entry whereas chronic hyperglycaemia cause downregulation of ACE2 expression making the $\beta$ cell function vulnerable to the inflammatory and damaging effect of the virus. ${ }^{2}$

People with diabetes who have not been infected with the SARS-CoV-2 virus should intensify their metabolic control as needed as means of primary prevention of COVID-19 which includes continuation and adequate control of blood pressure and lipids. Wherever possible, remote consultations using Connected Health models should be utilised to reduce exposure. They should also be encouraged

Corresponding Author:

Dr. Robin Maskey, Additional Professor, Department of Internal Medicine, B.P.Koirala Institute of Health Sciences, Dharan, Nepal. to follow general advice from $\mathrm{WHO}$, the $\mathrm{CDC}$, and local governments about hand washing and physical distancing.

It has been recommended to continue with their antihypertensive regimens including ACE inhibitors and angiotensin 2 receptors. Statins have been shown to restore the reduction of ACE2 induced by high lipids such as low density lipoprotein or lipoprotein(a). ${ }^{3}$ That's why statins should not be discontinued because of the long-term benefits and the potential for tipping the balance towards a cytokine storm by rebound rises in interleukin(IL)-6 and IL- $1 \beta$.

There is increase in the prevalence of severe DKA in COVID-19 positive patients with type 1 diabetes but this might in part be because of delayed hospital admission. So, we have to make patients with type 1 diabetes aware of this complication and educate about typical symptoms, home measurement of urine or blood ketones, acute behaviour guidelines, and liberal and early inquiry of professional medical advice and sick day rules.

Patients with type 2 diabetes and fatty liver disease have an increased risk for cytokine storm, and such patients should be considered at increased risk of severe COVID-19 disease and screen for hyperinflammation markers (eg; increasing ferritin, decreasing platelet counts, high-sensitivity C-reactive protein, or erythrocyte sedimentation rate).

Patients with obesity and diabetes are at risk of ventilatory failure and complications during mechanical ventilation. Obesity impairs 
immune response against microbial agents including macrophage activation and inhibition of pro-inflammatory cytokines and leads to a dysregulation of the immune response contributing o complications associated with obesity. ${ }^{4}$

Lactic acidosis associated with metformin, or euglycaemic or moderate hyperglycaemic diabetic ketoacidosis associated with SGLT-2 inhibitors so these drugs should be discontinued for patients with severe symptoms of COVID-19 to reduce the risk of acute metabolic decompensation.DPP-4 inhibitors should be discontinued. If frequent occurrence of diarrhoea and sepsis all OHA are discontinued and managed by intravenous infusion..$^{5 \text { and } 6}$

Executing clinical trials and evidence-based therapies under challenging circumstances has been proven feasible during the COVID-19 pandemic. $^{7}$ Investigating subgroups with diabetes and how these relate and managing diabetes to COVID-19 outcomes will be important. ${ }^{8}$

\section{References}

1.Chen Y, Gong X, Wang L, Guo J. Effects of hypertension, diabetes and coronary heart disease on COVID-19 diseases severity:[SEP:a systematic review and meta-analysis

2. Yang JK, Lin SS, Ji XJ, Guo LM. Binding of SARS coronavirus to its receptor damages islets and causes acute diabetes. Acta Diabetol 2010; 47: 193-99.

3.Shin YH, Min JJ, Lee JH. The effect of fluvastatin on cardiac fibrosis and angiotensin-converting enzyme-2 expression in glucose-controlled diabetic rat hearts.

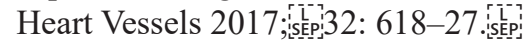

4.Luzi L, Radaelli MG. Influenza and obesity: its odd relationship and the lessons for COVID-19 pandemic. Acta Diabetol 2020;

5.Hartmann-Boyce J, Morris E, Goyder C, et al. Managing diabetes during the COVID- 19 epidemic. 2020.

6. campaign: international guidelines for management of severe sepsis and septic shock: 2012. Critical Care Med 2013; 41: 580-637.

7. Cao B, Wang Y, Wen D, et al. A trial of lopinavirritonavir in adults hospitalized with severe Covid-19. N Engl J Med 2020; published online March 18. DOI:10.1056/NEJMoa2001282. SEEP:

8. Wondafrash DZ, Desalegn TZ, Yimer EM. Potential effect of hydroxychloroquine in diabetes mellitus: a systematic review on preclinical and clinical trial studies. J Diabetes Res 2020; sêpia 2020: 5214751. 